\title{
COLLAPSING ALONG MONOTONE POSET MAPS
}

\author{
DMITRY N. KOZLOV
}

Received 21 March 2005; Revised 13 September 2005; Accepted 12 February 2006

We introduce the notion of nonevasive reduction and show that for any monotone poset map $\varphi: P \rightarrow P$, the simplicial complex $\Delta(P)$ NE-reduces to $\Delta(Q)$, for any $Q \supseteq \operatorname{Fix} \varphi$.

As a corollary, we prove that for any order-preserving map $\varphi: P \rightarrow P$ satisfying $\varphi(x) \geq$ $x$, for any $x \in P$, the simplicial complex $\Delta(P)$ collapses to $\Delta(\varphi(P))$. We also obtain a generalization of Crapo's closure theorem.

Copyright (c) 2006 Hindawi Publishing Corporation. All rights reserved.

\section{Order complexes, collapsing, and NE-reduction}

For a poset $P$ we let $\Delta(P)$ denote its nerve: the simplicial complex whose simplices are all chains of $P$. For a simplicial complex $X$ we let $V(X)$ denote the set of its vertices.

An elementary collapse in a simplicial complex $X$ is a removal of two open simplices $\sigma$ and $\tau$ from $X$, such that $\operatorname{dim} \sigma=\operatorname{dim} \tau+1$, and $\sigma$ is the only simplex of $X$, different from $\tau$ itself, which contains the simplex $\tau$ in its closure.

When $Y$ is a subcomplex of $X$, we say that $X$ collapses onto $Y$ if there exists a sequence of elementary collapses leading from $X$ to $Y$; in this case we write $X \searrow Y$ (or, equivalently, $Y \nearrow X)$.

Definition 1.1. (1) A finite nonempty simplicial complex $X$ is called nonevasive if either $X$ is a point, or, inductively, there exists a vertex $v$ of $X$, such that both $X \backslash\{v\}$ and $\operatorname{lk}_{X} v$ are nonevasive.

(2) For two nonempty simplicial complexes $X$ and $Y$, write $X \searrow_{\mathrm{NE}} Y$ (or, equivalently, $\left.Y{ }^{\prime} \mathrm{NE} X\right)$, if there exists a sequence $X=A_{1} \supset A_{2} \supset \cdots \supset A_{t}=Y$, such that for all $i \in$ $\{1, \ldots, t-1\}, V\left(A_{i}\right)=V\left(A_{i+1}\right) \cup\left\{x_{i}\right\}$, so that $l k_{A_{i}} x_{i}$ is nonevasive.

We recall that the notion of nonevasive simplicial complexes was introduced in [9], and was initially motivated by the complexity-theoretic considerations. For further connections to topology and more facts on nonevasiveness we refer to $[13,16]$. Recently an interesting connection has been established between discrete Morse theory and evasiveness, the standard references are $[7,8]$. 
Several classes of simplicial complexes are known to be nonevasive. Perhaps the simplest example is provided by the fact that all cones are nonevasive. A more complicated family of nonevasive simplicial complexes is obtained by taking the order complexes of the noncomplemented lattices, see [10].

In the situation described in Definition 1.1(2), we say that the simplicial complex $X$ NE-reduces to its subcomplex $Y$. The following facts about NE-reduction are useful for our arguments.

Fact 1. If $X_{1}$ and $X_{2}$ are simplicial complexes, such that $X_{1} \searrow_{\mathrm{NE}} X_{2}$, and $Y$ is an arbitrary simplicial complex, then $X_{1} * Y \searrow_{\mathrm{NE}} X_{2} * Y$.

Here the symbol $*$ denotes the simplicial join of two simplicial complexes, see [14]. That $X_{1} * Y \searrow_{\mathrm{NE}} X_{2} * Y$ follows by induction from the facts that if $v$ is any vertex of a simplicial complex $X$, then we have $\operatorname{lk}_{X * Y} v=\left(\operatorname{lk}_{X} v\right) * Y$ and $(X * Y) \backslash\{v\}=(X \backslash\{v\}) *$ $Y$, for any $v \in V(X)$.

To NE-reduce $X_{1} * Y$ to $X_{2} * Y$, simply take the sequence of vertices $x_{1}, \ldots, x_{t} \in V\left(X_{1}\right)$ which NE-reduces $X_{1}$ to $X_{2}$. We have $\operatorname{lk}_{X_{1} * Y}\left(x_{1}\right)=\left(\operatorname{lk}_{X_{1}}\left(x_{1}\right)\right) * Y$. In turn, the simplicial complex $\left(\operatorname{lk}_{X_{1}}\left(x_{1}\right)\right) * Y$ is nonevasive: this is seen by induction on the number of vertices of the first factor, with the base given by the fact that all cones are nonevasive. Removing $x_{1}$ from $X_{1} * Y$ yields the simplicial complex $\left(X_{1} \backslash\left\{x_{1}\right\}\right) * Y$, hence, continuing in this way, we will NE-reduce $X_{1} * Y$ all the way to $X_{2} * Y$.

Fact 2. The reduction $X \searrow_{\mathrm{NE}} Y$ implies $X \searrow Y$, which in turn implies that $Y$ is a strong deformation retract of $X$.

\section{Monotone poset maps}

Next we define a class of maps which are particularly suitable for our purposes.

Definition 2.1. Let $P$ be a poset. An order-preserving map $\varphi: P \rightarrow P$ is called a monotone map, if for any $x \in P$ either $x \geq \varphi(x)$ or $x \leq \varphi(x)$.

If $x \geq \varphi(x)$ for all $x \in P$, then $\varphi$ is called a decreasing map, analogously, if $x \leq \varphi(x)$ for all $x \in P$, then $\varphi$ is called an increasing map.

We remark here the fact that while a composition of two decreasing maps is again a decreasing map, and, in the same way, a composition of two increasing maps is again an increasing map, the composition of two monotone maps is not necessarily a monotone map.

Example 2.2. Let $P$ be the lattice of all subsets of $\{1,2\}$, and define $\varphi(S)=S \cup\{2\}$, and $\gamma(T)=T \backslash\{1\}$, for all $S, T \subseteq\{1,2\}$. The composition $T \circ S$ maps all the subsets to $\{2\}$, in particular it is not a monotone map.

On the other hand, any power of a monotone map is again monotone. Indeed, let $\varphi$ : $P \rightarrow P$ be monotone, let $x \in P$, and say $x \leq \varphi(x)$. Since $\varphi$ is order-preserving, we conclude that $\varphi(x) \leq \varphi^{2}(x), \varphi^{2}(x) \leq \varphi^{3}(x)$, and so forth. Hence $x \leq \varphi^{N}(x)$ for arbitrary $N$. 
The following proposition shows that monotone maps have a canonical decomposition in terms of increasing and decreasing maps.

Proposition 2.3. Let $P$ be a poset, and let $\varphi: P \rightarrow P$ be a monotone map. There exist unique maps $\alpha, \beta: P \rightarrow P$, such that

(i) $\varphi=\alpha \circ \beta$;

(ii) $\alpha$ is an increasing map, whereas $\beta$ is a decreasing map;

(iii) Fix $\alpha \cup \operatorname{Fix} \beta=P$.

Proof. Set

$$
\begin{aligned}
& \alpha(x):= \begin{cases}\varphi(x), & \text { if } \varphi(x)>x, \\
x, & \text { otherwise }\end{cases} \\
& \beta(x):= \begin{cases}\varphi(x), & \text { if } \varphi(x)<x, \\
x, & \text { otherwise. }\end{cases}
\end{aligned}
$$

Clearly, Fix $\alpha \cup \operatorname{Fix} \beta=P$. Let us see that $\varphi(x)=\alpha(\beta(x))$, for any $x \in P$. This is obvious if $\varphi(x) \geq x$, since then $\alpha(\beta(x))=\alpha(x)=\varphi(x)$ by (2.1) and (2.2), respectively. Assume $\varphi(x)<x$, then $\beta(x)=\varphi(x)$, hence $\alpha(\beta(x))=\alpha(\varphi(x))$. Since $\varphi$ is order-preserving, $\varphi(x)<x$ implies $\varphi(\varphi(x)) \leq \varphi(x)$. Thus (2.1) gives $\varphi(x) \in$ Fix $\alpha$, and we conclude that $\alpha(\beta(x))=$ $\alpha(\varphi(x))=\varphi(x)$.

To see that $\alpha$ is an increasing map, we just need to see that it is order-preserving. Since $\alpha$ either fixes an element or maps it to a larger one, the only situation which needs to be considered is when $x, y \in P, x<y$, and $\alpha(x)=\varphi(x)$. However, under these conditions we have $\alpha(y) \geq \varphi(y) \geq \varphi(x)=\alpha(x)$, and so $\alpha$ is order-preserving. That $\beta$ is a decreasing map can be seen analogously. Finally, the uniqueness follows from the fact that each $x \in P$ must be fixed by either $\alpha$ or $\beta$, and the value $\varphi(x)$ determines which one will fix $x$.

\section{The main theorem and implications}

Prior to this work, it has been known that a monotone map $\varphi: P \rightarrow P$ induces a homotopy equivalence between $\Delta(P)$ and $\Delta(\varphi(P))$, see [4, Corollary 10.17]. It was also proved in [4] that if the map $\varphi$ satisfies the additional condition $\varphi^{2}=\varphi$, then $\varphi$ induces a strong deformation retraction from $\Delta(P)$ to $\Delta(\varphi(P))$.

The latter result was strengthened in [11, Theorem 2.1], where it was shown that whenever $\varphi$ is an ascending (or descending) closure operator, $\Delta(P)$ collapses onto $\Delta(\varphi(P))$. There this fact was used to analyze the effect of the folding operation on the corresponding Hom complexes, see also [1-3, 12].

The next theorem strengthens and generalizes the results from $[4,11]$.

Theorem 3.1. Let $P$ be a poset, and let $\varphi: P \rightarrow P$ be a monotone map. Assume $P \supseteq Q \supseteq$ $\operatorname{Fix} \varphi, P \backslash Q$ is finite, and, for every $x \in P \backslash Q, P_{<x} \cup P_{>x}$ is finite, then $\Delta(P) \searrow_{\mathrm{NE}} \Delta(Q)$, in particular, $\Delta(P)$ collapses onto $\Delta(Q)$. 
Remarks 3.2. (1) Note that when $P$ is finite, the conditions of the Theorem 3.1 simply reduce to: $P \supseteq Q \supseteq \operatorname{Fix} \varphi$.

(2) Under conditions of Theorem 3.1, the simplicial complex $\Delta(P)$ collapses onto the simplicial complex $\Delta(Q)$, in particular, the complexes $\Delta(P)$ and $\Delta(Q)$ have the same simple homotopy type, see [5].

(3) Under conditions of Theorem 3.1, the topological space $\Delta(Q)$ is a strong deformation retract of the topological space $\Delta(P)$.

(4) Any poset $Q$ satisfying $P \supseteq Q \supseteq \varphi(P)$ will also satisfy $P \supseteq Q \supseteq \operatorname{Fix} \varphi$, hence Theorem 3.1 will apply. In particular, for finite $P$, we have the following corollary.

Corollary 3.3. For any poset $P$, and for any monotone map $\varphi: P \rightarrow P$, satisfying conditions of Theorem 3.1, $\Delta(P) \searrow_{\mathrm{NE}} \Delta(\varphi(P))$.

It is easy to prove Theorem 3.1, once the following auxiliary result is established.

Proposition 3.4. Let $P$ be a poset, and let $\varphi: P \rightarrow P$ be a monotone map. Assume $x \in$ $P$, such that $\varphi(x) \neq x$, and $P_{<x} \cup P_{>x}$ is finite, then $\Delta\left(P_{<x}\right) * \Delta\left(P_{>x}\right)$ is nonevasive. More precisely, if $\varphi(x)<x$, then $\Delta\left(P_{<x}\right)$ is nonevasive, and if $\varphi(x)>x$, then $\Delta\left(P_{>x}\right)$ is nonevasive.

Proof. Since the expression $\Delta\left(P_{<x}\right) * \Delta\left(P_{>x}\right)$ is symmetric with respect to inverting the partial order of $P$, it is enough, without loss of generality, to only consider the case $\varphi(x)<$ $x$. Let us show that in this case $\Delta\left(P_{<x}\right)$ is nonevasive. We proceed by induction on $\left|P_{<x}\right|$. If $\left|P_{<x}\right|=1$, then the statement is clear, so assume $\left|P_{<x}\right| \geq 2$.

Let $\psi: P_{<x} \rightarrow P_{<x}$ denote the restriction of $\varphi$. It is easy to see that $\psi$ is a monotone map of $P_{<x}$. To verify that $\Delta\left(P_{<x}\right) \searrow_{\mathrm{NE}} \Delta\left(P_{\leq \varphi(x)}\right)$, order the elements of $P_{<x} \backslash P_{\leq \varphi(x)}$ following an arbitrary linear extension in the decreasing order, say $P_{<x} \backslash P_{\leq \varphi(x)}=\left\{a_{1}, \ldots, a_{t}\right\}$, and $a_{i} \nless a_{j}$, for $i<j$. By the choice of the order of $a_{i}$ 's, we have $P_{<a_{i}}=P_{<a_{i}}^{i}$, where $P^{i}=P \backslash$ $\left\{a_{1}, \ldots, a_{i-1}\right\}$. Therefore, by the induction assumption, $\Delta\left(P_{<a_{i}}\right)$ is nonevasive for all $1 \leq$ $i \leq t$, and we have

$$
\Delta\left(P_{<x}\right)=\Delta\left(P_{<x}^{1}\right) \searrow_{\mathrm{NE}} \Delta\left(P_{<x}^{2}\right) \searrow_{\mathrm{NE}} \cdots \searrow_{\mathrm{NE}} \Delta\left(P_{<x}^{t+1}\right)=\Delta\left(P_{\leq \varphi(x)}\right)
$$

On the other hand, $\Delta\left(P_{\leq \varphi(x)}\right)$ is a cone, hence it is nonevasive, and therefore $\Delta\left(P_{<x}\right)$ is nonevasive as well. It follows that $\Delta\left(P_{<x}\right) * \Delta\left(P_{>x}\right)$ is nonevasive.

Proof of Theorem 3.1. The proof is by induction on $|P \backslash Q|$. The statement is trivial when $|P \backslash Q|=0$, so assume $|P \backslash Q| \geq 1$.

To start with, we replace the monotone map $\varphi$ with a monotone map $\gamma$ satisfying $\gamma(P) \subseteq Q$ and $\operatorname{Fix} \gamma=\operatorname{Fix} \varphi$. To achieve that objective we can set $\gamma:=\varphi^{N}$, where $N=|P|$ $Q \mid$. With this choice of $\gamma$, the inclusion $\gamma(P) \subseteq Q$ follows from the assumption that $\operatorname{Fix} \varphi \subseteq$ $Q$, since Fix $\gamma=\gamma(P)$.

Take arbitrary $x \in P \backslash Q$. Since $x \notin \gamma(P)$, we have $x \neq \gamma(x)$, hence by Proposition 3.4 we know that $\operatorname{lk}_{\Delta(P)} x=\Delta\left(P_{<x}\right) * \Delta\left(P_{>x}\right)$ is nonevasive. This means $\Delta(P) \searrow_{\mathrm{NE}} \Delta(P \backslash\{x\})$.

Let $\psi: P \backslash\{x\} \rightarrow P \backslash\{x\}$ be the restriction of $\gamma$. Clearly, $\psi$ is a monotone map, and Fix $\psi=$ Fix $\gamma$. This implies that Fix $\psi \subseteq Q$, hence, by the induction hypothesis $\Delta(P \backslash\{x\})$ $\searrow_{\mathrm{NE}} \Delta(Q)$. Summarizing, we conclude that $\Delta(P) \searrow_{\mathrm{NE}} \Delta(Q)$. 
On the enumerative side, we obtain the following generalization of Crapo's closure theorem from 1968, see [6, Theorem 1].

Corollary 3.5. Let $P$ be a finite poset with $\hat{0}$ and $\hat{1}$, and let $\varphi: P \rightarrow P$ be an increasing map. Assume $P \supseteq Q \supseteq \operatorname{Fix} \varphi$, and $Q \cap \varphi^{-\infty}(\hat{1})=\{\hat{1}\}$. Then,

$$
\sum_{\varphi^{\infty}(z)=\hat{1}} \mu_{P}(\hat{0}, z)= \begin{cases}\mu_{Q}(\hat{0}, \hat{1}), & \text { if } \hat{0} \in \operatorname{Fix} \varphi \\ 0, & \text { otherwise. }\end{cases}
$$

Here $\varphi^{\infty}$ is the stabilization of $\varphi$, say $\varphi^{\infty}:=\varphi^{|P|}$, so $\varphi^{-\infty}(\hat{1})$ denotes the set of all elements of $P$ which map to $\hat{1}$ after a sufficiently high iteration of $\varphi$.

Before we give the proof, recall the following convention: whenever $P$ is a poset with $\hat{0}$ and $\hat{1}$, we let $\bar{P}$ denote $P \backslash\{\hat{0}, \hat{1}\}$.

Proof of Corollary 3.5. Assume first that $\hat{0} \in \operatorname{Fix} \varphi$, hence $\hat{0}, \hat{1} \in Q$, and, since $\varphi$ is increasing, $\varphi^{-\infty}(\hat{0})=\{\hat{0}\}$. Set $R:=\left(P \backslash \varphi^{-\infty}(\hat{1})\right) \cup\{\hat{0}, \hat{1}\}$, that is, $R$ is the set of all elements of $\bar{P}$ which will not map to $\hat{1}$, no matter how high iteration of $\varphi$ we take, with the maximal and the minimal elements attached. Let $\psi: \bar{R} \rightarrow \bar{R}$ be the restriction of $\varphi$. Clearly, $\psi$ is a monotone map, and Fix $\psi=\operatorname{Fix} \varphi \backslash\{\hat{0}, \hat{1}\}$.

Since $\bar{R} \supseteq \bar{Q} \supseteq$ Fix $\psi$, we conclude that $\Delta(\bar{R})$ collapses onto $\Delta(\bar{Q})$; in particular the simplicial complexes $\Delta(\bar{R})$ and $\Delta(\bar{Q})$ have the same Euler characteristic. By Ph. Hall's theorem, see [15], for any poset $P$ with a maximal element and a minimal element we have $\chi(\Delta(\bar{P}))=\mu_{P}(\hat{0}, \hat{1})$, therefore here we conclude that $\mu_{Q}(\hat{0}, \hat{1})=\mu_{R}(\hat{0}, \hat{1})$.

On the other hand, by definition of the Möbius function, we have the equality $\sum_{z \in P} \mu_{P}(\hat{0}, z)=0$, which can be rewritten as $\sum_{z \in \varphi^{-\infty}(\hat{1})} \mu_{P}(\hat{0}, z)=-\sum_{z \notin \varphi^{-\infty}(\hat{1})} \mu_{P}(\hat{0}, z)$. Similarly, $\mu_{R}(\hat{0}, \hat{1})=-\sum_{x \in R \backslash\{\hat{1}\}}(\hat{0}, x)$. Since the condition $z \notin \varphi^{-\infty}(\hat{1})$ is equivalent to the condition $z \in R \backslash\{\hat{1}\}$, and $R_{<x}=P_{<x}$, for any $x \in R$, we conclude that $\mu_{Q}(\hat{0}, \hat{1})=\mu_{R}(\hat{0}, \hat{1})=$ $\sum_{z \in \varphi^{-\infty}(\hat{1})} \mu_{P}(\hat{0}, z)$.

Consider now the case $\hat{0} \notin \operatorname{Fix} \varphi$. If $\varphi^{\infty}(\hat{0})=\hat{1}$, then the statement follows from the definition of the Möbius function, since then $\varphi^{\infty}(z)=\hat{1}$ for all $z \in P$. Assume $\varphi^{\infty}(\hat{0}) \neq \hat{1}$. We can define a new map $\psi$ by changing the value of $\varphi$ in one element:

$$
\psi(x)= \begin{cases}\hat{0}, & \text { if } x=\hat{0} \\ \varphi(x), & \text { otherwise }\end{cases}
$$

Clearly, $\psi$ is a monotone function, $\operatorname{Fix} \psi=\operatorname{Fix} \varphi \cup\{\hat{0}\}$, and $\varphi^{-\infty}(\hat{1})=\psi^{-\infty}(\hat{1})$. Hence, the first part of the proof applies, and we conclude that

$$
\sum_{\psi^{\infty}(z)=\hat{1}} \mu_{P}(\hat{0}, z)=\sum_{\varphi^{\infty}(z)=\hat{1}} \mu_{P}(\hat{0}, z)=\mu_{Q}(\hat{0}, \hat{1})
$$




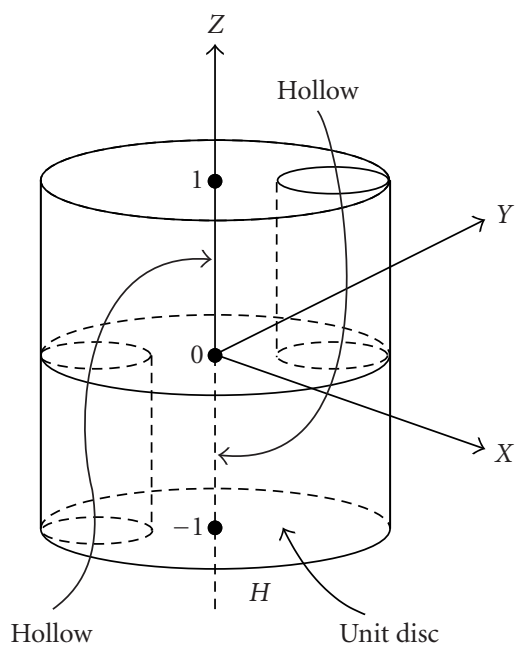

Figure 4.1. A house with two rooms.

for any $Q$ such that $P \supseteq Q \supseteq$ Fix $\psi$, and such that $Q \cap \varphi^{-\infty}(\hat{1})=\{\hat{1}\}$. Choose $Q=\left(P_{\geq \varphi(\hat{0})} \backslash\right.$ $\left.\varphi^{-\infty}(\hat{1})\right) \cup\{\hat{0}, \hat{1}\}$. Since $\varphi(\hat{0}) \notin \varphi^{-\infty}(\hat{1})$, the poset $Q$ has only one atom $\varphi(\hat{0})$, thus we have $\mu_{Q}(\hat{0}, \hat{1})=0$, and the proof is complete.

\section{NE-reduction and collapses}

The NE-reduction can be used to define an interesting equivalence relation on the set of all simplicial complexes.

Definition 4.1. Let $X$ and $Y$ be simplicial complexes. Recursively, it is said that $X \simeq_{\mathrm{NE}} Y$ if $X \succ_{\mathrm{NE}} Y$, or $Y{ }^{\prime}{ }_{\mathrm{NE}} X$, or if there exists a simplicial complex $Z$, such that $X \simeq_{\mathrm{NE}} Z$ and $Y \simeq_{\mathrm{NE}} Z$.

Clearly, if $X$ is nonevasive, then $X \simeq_{\mathrm{NE}} \mathrm{pt}$, but is the opposite true? The answer to that is "no." To see this, consider the standard example of a space which is contractible, but not collapsible: let $H$ be the so-called house with two rooms, see Figure 4.1.

The space $H$ is not collapsible, hence nonevasive, see [5] for an argument. On the other hand, we leave it to the reader to see that it is possible to triangulate the filled cylinder $C$ given by the equations $|z| \leq 1, x^{2}+y^{2} \leq 1$, so that $C>_{\mathrm{NE}} H$.

The analogous equivalence relation, where $\succ_{\mathrm{NE}}$ and $\nearrow_{\mathrm{NE}}$ are replaced by $\searrow$ and $\nearrow$, is called the simple homotopy equivalence; its equivalence classes are called simple homotopy types. The celebrated theorem of J.H.C. Whitehead states that the simplicial complexes with the simple homotopy type of a point are precisely those which are contractible, see [5]. Therefore, the class of the simplicial complexes which are NE-equivalent to a point relates to nonevasiveness in the same way as contractibility refers to collapsibility. Clearly, this means that this class should constitute an interesting object of study. 
We conjecture that the NE-equivalence is much finer than the Whitehead's simple homotopy type. We make two conjectures: a weak conjecture and a strong one.

Conjecture 4.2. There exist finite simplicial complexes $X$ and $Y$ having the same simple homotopy type, such that $X \neq_{\mathrm{NE}} Y$.

Conjecture 4.3. There exists an infinite family of finite simplicial complexes $\left\{X_{i}\right\}_{i=1}^{\infty}$, which all have the same simple homotopy type, such that $X_{i} \neq_{\mathrm{NE}} X_{j}$, for all $i \neq j$.

Again, in the simple homotopy setting, the phenomenon of the Conjectures 4.2 and 4.3 is governed by an algebraic invariant called the Whitehead torsion, namely: a homotopy equivalence between finite connected CW-complexes is simple if and only if its Whitehead torsion is trivial, see $[5,(22.2)]$. It is enticing to hope for an existence of some similar invariant in our NE-setting.

Finally, let us remark that whenever we have simplicial complexes $X \simeq_{\mathrm{NE}} Y$, there exists a simplicial complex $Z$, such that $X \nearrow_{\mathrm{NE}} Z \searrow_{\mathrm{NE}} Y$. Indeed, assume $A \searrow_{\mathrm{NE}} B{ }{ }_{\mathrm{NE}} C$, for some simplicial complexes $A, B$, and $C$. Let $S=V(A) \backslash V(B)$, and $T=V(C) \backslash V(B)$. Let $D$ be the simplicial complex obtained by attaching to $A$ the vertices from $T$ in the same way as they would be attached to $B \subseteq A$. Clearly, since the links of the vertices from $S$ did not change, they can still be removed in the same fashion as before, and therefore we have $A r_{\mathrm{NE}} D \searrow_{\mathrm{NE}} C$. Repeating this operation several times and using the fact that the reductions $\nearrow_{\mathrm{NE}}$ (as well as $\searrow_{\mathrm{NE}}$ ) compose, we prove the claim.

\section{Acknowledgments}

We would like to thank the Swiss National Science Foundation and ETH-Zürich for the financial support of this research. We also thank the referee for several valuable comments. This research is supported by Swiss National Science Foundation Grant PP002-102738/1.

\section{References}

[1] E. Babson and D. N. Kozlov, Topological obstructions to graph colorings, Electronic Research Announcements of the American Mathematical Society 9 (2003), 61-68.

[2] _ Complexes of graph homomorphisms, Israel Journal of Mathematics 152 (2006), 285312.

[3] _ Proof of the Lovász Conjecture, to appear in Annals of Mathematics. Second Series, http://arxiv.org/abs/math.CO/0402395.

[4] A. Björner, Topological methods, Handbook of Combinatorics, Vol. 1, 2 (R. Graham, M. Grötschel, and L. Lovász, eds.), Elsevier, Amsterdam, 1995, pp. 1819-1872.

[5] M. M. Cohen, A Course in Simple-Homotopy Theory, Graduate Texts in Mathematics, vol. 10, Springer, New York, 1973.

[6] H. H. Crapo, Möbius inversion in lattices, Archiv der Mathematik (Basel) 19 (1968), no. 6, 595607 (1969).

[7] R. Forman, Morse theory for cell complexes, Advances in Mathematics 134 (1998), no. 1, 90-145.

[8] Morse theory and evasiveness, Combinatorica 20 (2000), no. 4, 489-504.

[9] J. Kahn, M. Saks, and D. Sturtevant, A topological approach to evasiveness, Combinatorica 4 (1984), no. 4, 297-306. 
[10] D. N. Kozlov, Order complexes of noncomplemented lattices are nonevasive, Proceedings of the American Mathematical Society 126 (1998), no. 12, 3461-3465.

[11] A simple proof for folds on both sides in complexes of graph homomorphisms, Proceedings of the American Mathematical Society 134 (2006), no. 5, 1265-1270.

[12] Chromatic numbers, morphism complexes, and Stiefel-Whitney characteristic classes, Geometric Combinatorics (E. Miller, V. Reiner, and B. Sturmfels, eds.), IAS/Park City Mathematics Series, vol. 14, American Mathematical Society, Rhode Island; Institute for Advanced Study (IAS), New Jersey, in press, http://arxiv.org/abs/math.AT/0505563.

[13] H. Kurzweil, A combinatorial technique for simplicial complexes and some applications to finite groups, Discrete Mathematics 82 (1990), no. 3, 263-278.

[14] J. R. Munkres, Elements of Algebraic Topology, Addison-Wesley, California, 1984.

[15] R. P. Stanley, Enumerative Combinatorics. Vol. 1, Cambridge Studies in Advanced Mathematics, vol. 49, Cambridge University Press, Cambridge, 1997, corrected reprint of the 1986 original.

[16] V. Welker, Constructions preserving evasiveness and collapsibility, Discrete Mathematics 207 (1999), no. 1-3, 243-255.

Dmitry N. Kozlov: Institute of Theoretical Computer Science, Swiss Federal Institute of Technology

Zurich, 8092 Zurich, Switzerland

E-mail address: dkozlov@inf.ethz.ch 


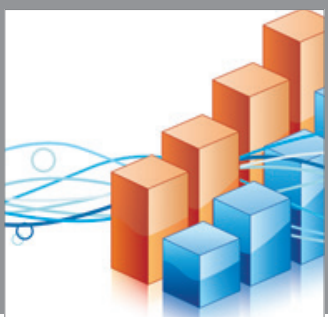

Advances in

Operations Research

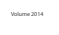

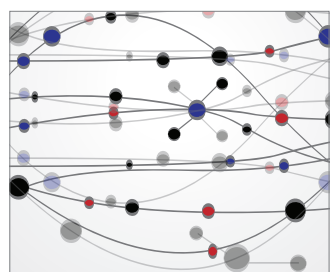

\section{The Scientific} World Journal
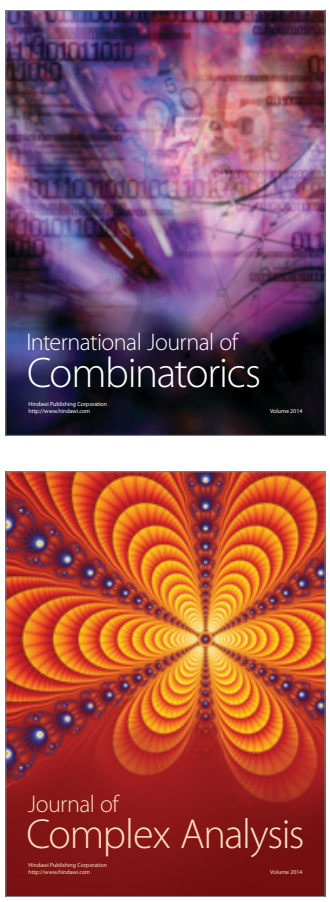

International Journal of

Mathematics and

Mathematical

Sciences
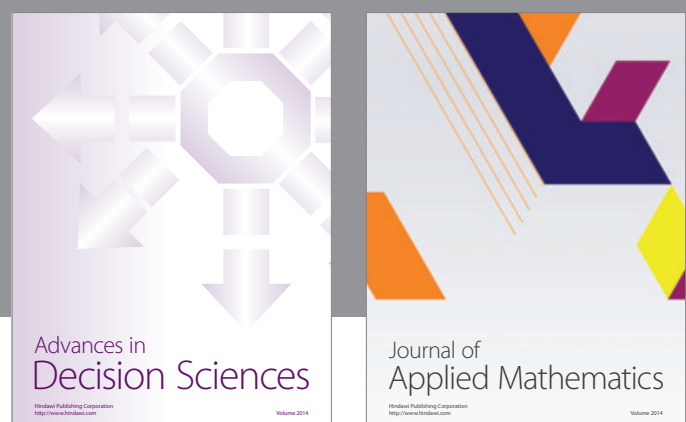

Journal of

Applied Mathematics
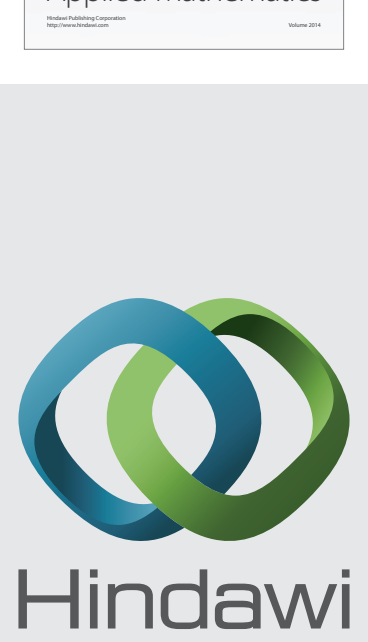

Submit your manuscripts at http://www.hindawi.com
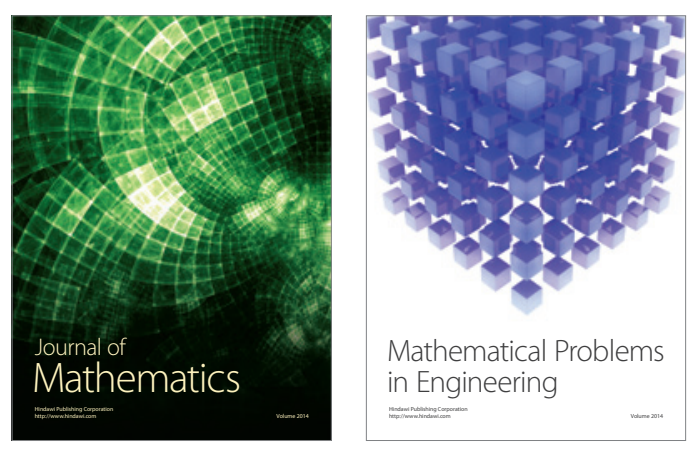

Mathematical Problems in Engineering
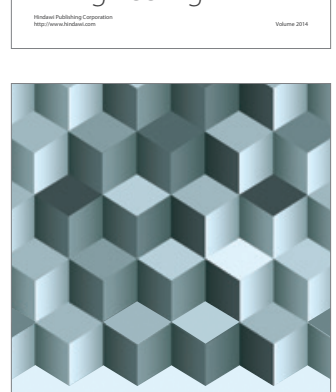

Journal of

Function Spaces
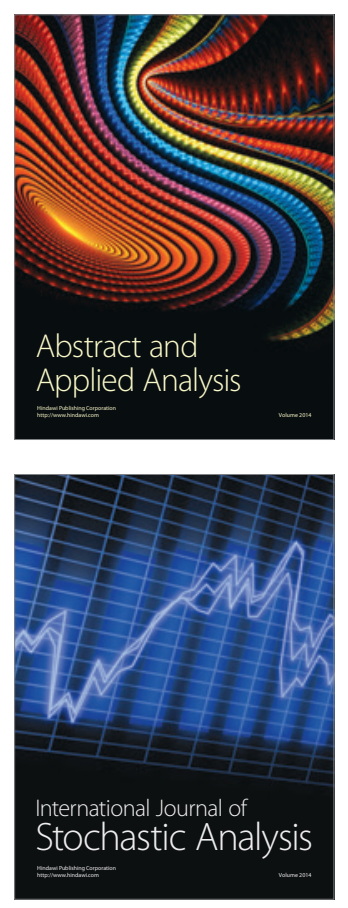

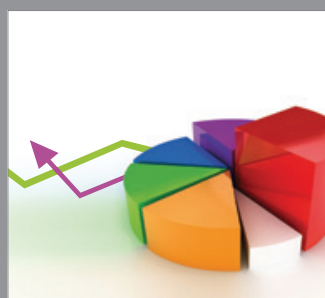

ournal of

Probability and Statistics

Promensencen
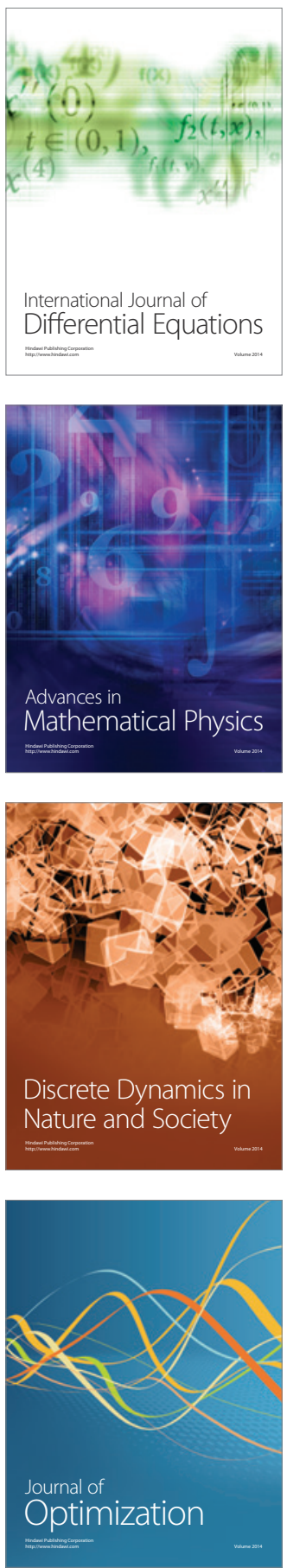